previously ${ }^{1}$ it was not included in this article. Kleihauer tests are performed at the majority of deliveries and any mother with a high feta count receives a larger dose of anti-D in accordance with the recommendation of the World Health Organisation.

In practice, however, I am not convinced that the matter of dose is a major factor in the incidence of failures of protection. For example, in the 35 cases of failure of protection in 1974-6 only three mothers had fetal counts above the 100 cells $/ 50$ low-power field level at which a larger dose of anti-D is recommended. One mother had a count of 672 cells and received a 500-ug dose, while another had 628 fetal cells and received $400 \mu \mathrm{g}$ of anti-D. The third had 375 fetal cells and this patient received a standard $100-\mu \mathrm{g}$ dose. Therefore if the fetal cell count/dose regimen had been adhered to one hundred per cent there would have only been one less case. It is my opinion that the major factors in the development of failures of protection are sensitisation prior to the anti-D administration and the ( ?genetically controlled) immunological responsiveness of the mother.

D TOveY

$$
\begin{aligned}
& \text { Regional 'Transfusion Centre, } \\
& \text { Leeds } \\
& 1 \text { Tovey, L A D, Health Trends, 1976, 8, } 25 .
\end{aligned}
$$

\section{Syringe-transmitted hepatitis}

SIR,-Professor A J Zuckerman (2 September, p 696) writes that "it is imperative that an adequately sterilised or a disposable syringe and needle should be used for each individua patient" and I strongly support his authoritative statement. It is disappointing, to say the least, that any less careful technique should be contemplated at the present day almost 35 years after the study in military personnel undergoing intravenous treatment for syphili established the method of infection, epidemiology, and prevention of syringe-transmitted hepatitis. This definitive study was reported in $1945^{1}$ and amplified in the Watson lecture of the Royal Faculty of Physicians and Surgeons of Glasgow in February 1947.2 Other reports of serum hepatitis arising in persons having venepunctures in a diabetic clinic subjected to mass immunisation, receiving blood transfusion, or undergoing tattooing indicated that the risk of acquiring hepatitis $B$ infection was not confined to the syphilis clinic. While I understand the logistic attractions of a multidose-syringe technique, the seriousness of serum hepatitis $\mathbf{B}$ infection demands the use of a technique that excludes any risk of serum transmission and fully justifies Professor Zuckerman's unequivoca statement.

$S$ M LAIRD

\section{Royal Victoria Hospital,}

Royal Victoria Hospital,

\footnotetext{
1 Laird, S M, British fournal of Venereal Diseases, 1946, 22, 29.
${ }^{2}$ Laird, S M, Glasgow Medical fournal, 1947, 28, 199.
}

SIR,-Professor A J Zuckerman (2 September, p 696) advocates the use of a separate disposable syringe and needle for the mass BCG vaccination of schoolchildren and says that the evidence for a change in the current practice is indisputable. Several years of annual reports of the chief medical officer on the state of the public health in England show that some half million schoolchildren are vaccinated each year with BCG, and this will have been accomplished in the vast majority by using a separate syringe for every 10 injections and a new needle for each child. Statutory notification of infectious jaundice was introduced in 1968, but the literature of this sort of case occurring following BCG vaccination is non-existent.

Professor Zuckerman cites cases of hepatitis occurring in groups such as diabetics after venepuncture and Royal Navy personnel receiving intramuscular injections from a multidose syringe for syphilis and extrapolates the evidence so as to incriminate intradermal injections in school children. This is clearly not good enough, though there may be theoretical grounds to change the present practice so that each child receives vaccine from a separate disposable syringe and needle. The consequence of such a change would be a vastly increased cost for the Health Service in terms of both syringes and doctors' and nurses' time as the procedure would be slowed down. The extra cost would, of course, need to come from the finite pool of Health Service resources and I would like to query whether the increased costs are justifiable.

\section{HARVEY GORDON}

Mid-Surrey District, Surrey Area Health Authority, West Park Hospital, Epsom, Surrey

\section{Misdiagnosis of amoebiasis}

SIR,-It was surely inevitable that the amoebiasis unit should be reorganised following the retirement of its founder last year and we are sorry that this seems to have become the subject of controversy in your columns. Following in your footsteps (leading article, 5 August, p 379) and unreassured by our reply (26 August, p 632), Professor Brian Maegraith (9 September, p 769) regrets the "absorption" of the unit into the pathology department of the Hospital for Tropical Diseases. Despite rather gross disparity of resources, this department was already handling just three times as many routine investigations for amoebiasis as the then separate and wellpublicised amoebiasis unit. Since the merger the combined figures have continued to increase. The joint amoebiasis service is separately funded by the Public Health Laboratory Service. We know of no evidence that the reference services are inadequate and as Dr J E M Whitehead points out ( September, $p$ 769), there is no reason why anyone should be in ignorance of them; yet Air Vice-Marshal Stamm (9 September, p 769) sees a possible connection between the death of Dr T H Foley's patient (5 August, p 428) and the "loss of independence" of the amoebiasis unit. The death is reported to have occurred about two years ago, at a time when the unit was still under Air Vice-Marshal Stamm's direction.

D S RIDLEY

Hospital for Tropical Diseases, D C WARHURST

London NW

\section{Blood cultures for diagnosis of endocarditis}

SIR,-Dr Celia Oakley, in her valuable article "Use of antibiotics-endocarditis" (12 August, p 489), states that between four and six blood cultures should be taken over an hour or two before treatment is given. We believe that this is excessive. It imposes a burden on the laboratory, which will have to spend a disproportionate time on large numbers of negative cultures from patients suspected of, but not suffering from, endocarditis. It is also a burden on the patient.

There is good evidence that three cultures are sufficient. ${ }^{1}$ When one is positive all three will be positive in a very high proportion of cases and the need for more than one in endocarditis is to allow for accidental contamination during collection or in the laboratory and to confirm the diagnosis when growth fails to appear in all culture bottles from a single sample. More cultures may be needed from patients who have had previous infection, especially those with prostheses, in whom the diagnosis is less likely to be straightforward, but for primary diagnosis three is sufficient.

E JOAN STOKES A Hollman

University College Hospital,

University Coll
London $\mathrm{WC1}$

${ }^{1}$ Crowley, N, fournal of Clinical Pathology, 1970, 23, 166.

\section{Bell's palsy and herpes simplex}

SIR,-Dr John B Wilson's statement (2 September, p 704) that "these results demonstrate a definite relationship between Bell's palsy and herpes simplex infection or reinfection in two of these cases and support the concept that Bell's palsy is a disease of multifactorial aetiology" is highly dubious. No one would dispute that facial nerve palsy can be caused by a variety of insults; herpes zoster is a well-known viral cause. Zoster of the facial nerve is often associated with sensory zoster of branches of the trigeminal or upper cervical nerves.

Unfortunately the information on $\mathrm{Dr}$ Wilson's four patients is inadequate. It is a pity that no culture of the vesicle fluid of the "typical herpetiform rash around the auricle" was attempted; it might have revealed that the second patient had zoster. A varicella-zoster titre of $1 / 256$, not least in a 92 -year-old patient, in specimens taken 20 days after the onset of the rash and at about four weeks is consistent with recent zoster. It would have been most helpful to have had the zoster titres in all the patients, not just in case 2 . Case 1 might again have been one of facial zoster, and the rise in herpes simplex titre might have been anamnestic. We were given no information about whether these patients had a history of recurrent herpes simplex lesions. I find it impossible to explain the herpes simplex titres in case 2 . One assumes the tests were carried out at the same time. Cases 3 and 4 prove nothing, for the herpes simplex titres have not changed significantly. Radcliffe Infirmary,
Oxford

BENT JUEL-JENSEN

SIR,-Dr John Wilson (2 September, p 704) contends that in his four cases the serological results demonstrate a definite relationship between Bell's palsy and herpes simplex infection or reinfection. However, there are serological cross-reactions between herpes simplex and varicella-zoster ( $\mathrm{V}-\mathrm{Z})$, and rising antibody titres to herpes simplex may be 